\title{
Phase-sensitive Four-wave Mixing in AIGaAs-on-Insulator Nano-waveguides
}

Da Ros, Francesco; Pu, Minhao; Ottaviano, Luisa; Hu, Hao; Semenova, Elizaveta; Galili, Michael; Yvind, Kresten; Oxenløwe, Leif Katsuo

Published in:

Proceedings of 2016 IEEE Photonics Conference

Publication date:

2016

Document Version

Peer reviewed version

Link back to DTU Orbit

Citation (APA):

Da Ros, F., Pu, M., Ottaviano, L., Hu, H., Semenova, E., Galili, M., Yvind, K., \& Oxenløwe, L. K. (2016). Phasesensitive Four-wave Mixing in AIGaAs-on-Insulator Nano-waveguides. In Proceedings of 2016 IEEE Photonics Conference IEEE.

\section{General rights}

Copyright and moral rights for the publications made accessible in the public portal are retained by the authors and/or other copyright owners and it is a condition of accessing publications that users recognise and abide by the legal requirements associated with these rights.

- Users may download and print one copy of any publication from the public portal for the purpose of private study or research.

- You may not further distribute the material or use it for any profit-making activity or commercial gain

- You may freely distribute the URL identifying the publication in the public portal

If you believe that this document breaches copyright please contact us providing details, and we will remove access to the work immediately and investigate your claim 


\title{
Phase-sensitive Four-wave Mixing in AlGaAs-on-Insulator Nano-waveguides
}

\author{
F. Da Ros, M. Pu, L. Ottaviano, H. Hu, E. Semenova, M. Galili, K. Yvind, and L. K. Oxenløwe \\ DTU Fotonik, Technical University of Denmark. DK-2800 Kgs. Lyngby, Denmark. fdro@ fotonik.dtu.dk
}

\begin{abstract}
Phase-sensitive four-wave mixing is experimentally demonstrated in a 5-mm long AlGaAsOI nano-waveguide. More than $7 \mathrm{~dB}$ of phase-sensitive extinction ratio are reported without neither using active biasing nor polarization-assisted schemes. Measurements show a good match with numerical predictions.
\end{abstract}

Keywords-four-wave mixing; phase sensitive amplification; integrated waveguides

\section{INTRODUCTION}

Several applications of phase-sensitive processing have been reported in the recent years, spanning from low-noise amplitication $^{1}$, signal regeneration ${ }^{2}$ quadrature decomposition $^{3}$ and optical filtering ${ }^{4}$. These demonstrations have been achieved mainly using four-wave mixing (FWM) in highly nonlinear fibers. The use of more compact devices would enable not only to reduce the footprint and potentially the energy consumption but also enable more complex functionality thanks to a more effective control of the phase of the interacting waves.

Along this direction, very recently the first fully integrated phase-sensitive processor has been reported ${ }^{5}$ using semiconductor optical amplifiers (SOAs). Regardless of the impressive demonstration, the achievable phase-sensitive extinction ratio (PER) was still limited and SOAs may not be the most suited nonlinear medium as their speed is constrained by the carrier lifetime. Alternative media have also been investigated with very promising results reported in periodically poled lithium niobate ${ }^{6}$ (PPLN), silicon ${ }^{7,8}$ and silicon-germanium ${ }^{9}$ waveguides. While PPLN may be difficult to integrate, silicon and silicon-germanium are fully CMOS compatible. However, due to the presence of twophoton absorption (TPA) at telecom wavelengths, the limited conversion efficiency (CE) in silicon restricts the achievable PER. Techniques have been reported to tackle this limitation by either using $\mathrm{p}-\mathrm{i}-\mathrm{n}$ junctions to prevent free-carrier accumulation ${ }^{7}$ at the expenses of additional fabrication steps, or by moving into narrow-band slow-light regimes under pulsed operation ${ }^{8}$ or by using polarization-assisted schemes ${ }^{9}$ which however result in a significant loss of optical signal-to-noise ratio. Alternatively to silicon, AlGaAs-on-insulator (AlGaAsOI) is an efficient nonlinear platform where TPA at $1550 \mathrm{~nm}$ can be addressed by proper bandgap engineering ${ }^{10}$.

In this work, we report the first demonstration of phase-sensitive FWM in a 5-mm long AlGaAsOI nanowaveguide without any need for active biasing, slow-light effects or polarization-assisted schemes. PERs up to $7.7 \mathrm{~dB}$ are achieved in good match with numerical predictions.

\section{WAVEGUIDE FABRICATION AND PROPERTIES}

The AlGaAsOI wafer is prepared by wafer growth, wafer bonding and substrate removal. The choice of an AlGaAs core $(\mathrm{n} \approx 3.3$ ) surrounded by the insulator cladding $(\mathrm{n} \approx 1.5)$ significantly enhances the field confinement in the waveguide and thus the already strong nonlinear Kerr effects in $\mathrm{AlGaAs}^{10}\left(\mathrm{n}_{2} \approx 10^{-17} \mathrm{~W} / \mathrm{m}^{2}\right)$. The nanowaveguides are defined by electron-beam lithography and dry etching using hydrogen silsesquioxane as hard mask. The inset in Fig. 1(a) shows a SEM image of the waveguide prior to $\mathrm{SiO}_{2}$ over-cladding deposition. The cross-section of the $5-\mathrm{mm}$ long waveguide is $290 \times 630 \mathrm{~nm}^{2}$ and the propagation loss has been estimated to $1.5 \mathrm{~dB} / \mathrm{cm}$. In order to increase the coupling efficiency into the waveguide, inverse tapers ${ }^{11}$ and lensed fibers have been used at both facets with an estimated coupling loss of $2.5 \mathrm{~dB} / \mathrm{facet}$. The phase-insensitive output $\mathrm{CE}$, measured by coupling into the waveguide a strong continuous wave $(\mathrm{CW})$ pump together with a weak CW signal, is shown in Fig. 1(a) as a function of the total power at the input of the waveguide. The output $\mathrm{CE}$ is defined as the ratio between idler and signal power at the output of the waveguide and as can be seen it increases almost quadratically with the input power showing no sign of saturation due to nonlinear loss up to a $\mathrm{CE}$ of $-10 \mathrm{~dB}$.

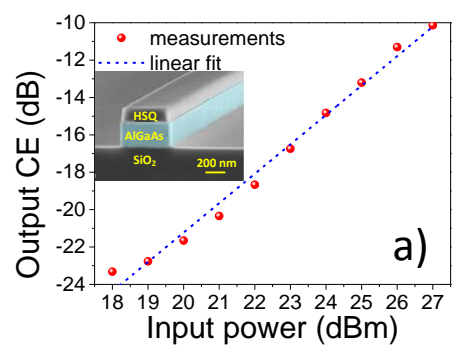

b) Freq. Comb Generator

Fig. 1. (a) Phase-insensitive output $\mathrm{CE}$ as a function of the input power for the 5-mm long AlGaAsOI waveguide: inset, SEM image of the nano-waveguide prior to PECVD deposition. (b) Experimental setup for phase-sensitive FWM characterization of the AlGaAsOI nano-waveguide. 

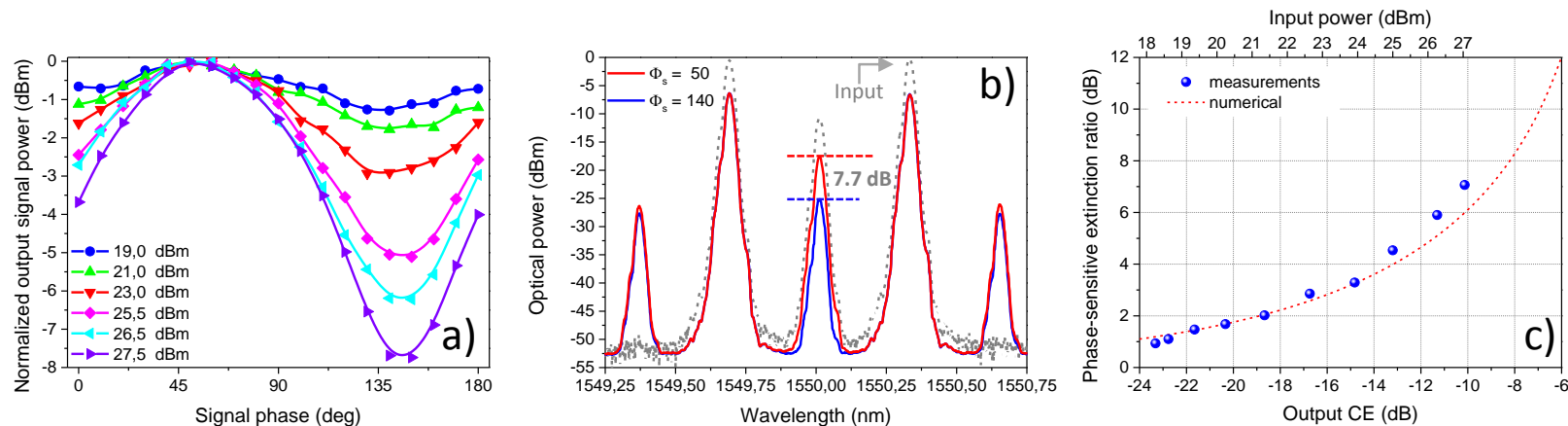

Fig. 2. Experimental results. (a) Normalized output signal power as a function of the input signal phase for different input power levels; (b) spectra at the input (dashed) and output (solid) of the waveguide for maximum amplification (red) and de-amplification (blue) for a total input power of 27.5 dBm; (c) phase-sensitive extinction ratio as a function of the phase-insensitive output CE: measurements (symbols) and numerical simulations (dashed line).

\section{EXPERIMENTAL SETUP}

The experimental setup is shown in Fig. 1 (b). A frequency comb is generated phase-modulating a $\mathrm{CW}$ wave at $1550 \mathrm{~nm}$ from an external cavity laser (ECL) with a $40-\mathrm{GHz}$ radio-frequency signal. The $40-\mathrm{GHz}$ spaced frequency lines are therefore locked in frequency and phase such that phasesensitive processes can occur. An optical processor (Finisar, WaveShaper) is then used to select three neighboring lines, the outer two to act as pumps and the middle one as signal. Amplitude and phase of the three waves is independently controlled with the processor such that the two pump waves are equalized in power and the signal-to-pump power ratio is set to $-10 \mathrm{~dB}$. An erbium-doped fiber amplifier (EDFA) is then used to amplify the three waves, followed by an optical bandpass filter (OBPF) to suppress out-of-band noise. The optical power coupled into the AlGaAsOI nano-waveguide is controlled by a variable optical attenuator (VOA) and the state-of-polarization is aligned to the TE mode of the waveguide. At the output of the waveguide, the spectra are recorded with an optical spectrum analyzer (OSA).

\section{RESULTS AND DISCUSSION}

The phase-sensitive response is investigated by measuring the signal power at the output of the waveguide as the input signal phase is varied with respect to the pumps by using the optical processor. The normalized output signal power as a function of the signal phase is shown in Fig. 2(a) for different input power levels into the AlGaAsOI waveguide. The curves clearly show the expected sine-square trend with PER values (defined as ratio between maximum and minimum output power) increasing with the input power into the waveguide. Input and output optical spectra for an input power of $27.5 \mathrm{dBm}$ are shown in Fig. 2(b). The output signal power is maximized when the signal phase is set to $50^{\circ}$, and minimized (maximum de-amplification) when an additional $90^{\circ}$ phase shift is added leading to a PER of $7.7 \mathrm{~dB}$. In Fig. 2(c), the measured PER values are then compared with numerical predictions as a function of the output CE since the phase-sensitive behavior relies on the interference between frequency degenerate signal and idler. The numerical simulations have been carried out by solving the nonlinear Schrödinger equation using the split-step Fourier method, thus accounting for the contribution of higher-order FWM processes taking place in the waveguide. A good agreement can be seen between numerical predictions and measurements, showing that a further increase in $\mathrm{CE}$, for example with the use of a longer waveguide, would enable PER values suitable for system applications.

\section{CONCLUSION}

We have characterized phase-sensitive FWM in a 5-mm long $\mathrm{AlGaAsOI}$ waveguide, reporting a PER above $7 \mathrm{~dB}$ without the need for active voltage biasing, slow light effects or polarization-assisted schemes. Comparison with numerical simulations shows the strong potential of this platform for implementing phase-sensitive processing in fully integrated devices.

\section{ACKNOWLEDGMENT}

This work was supported by the DNRF research center, SPOC, ref. DNRF123, the Villum Fonden center NATEC II and the SiMOF project.

\section{REFERENCES}

[1] Z. Tong, et al., "Towards ultrasensitive optical links enabled by lownoise phasesensitive amplifiers," Nat. Photonics 5, 430-436 (2011).

[2] R. Slavík, et al., "All-optical phase and amplitude regenerator for next-generation telecommunications systems," Nat. Photonics 4, 690-695 (2010).

[3] F. Da Ros, et al., "QPSK-to-2×BPSK wavelength and modulation format conversion through phase-sensitive four-wave mixing in a highly nonlinear optical fiber Opt. Express 21, 28743- 28750 (2013).

[4] J. Dailey, et al., "Optical Filtering Through Frequency Selective Phase-Sensitive Amplification and De-amplification," in Proc. OFC 2016, paper W4D.2.

[5] W. Li, et al., "First Demonstration of an Integrated Photonic PhaseSensitive Amplifier," in Proc. CLEO 2015, paper SW4N.5.

[6] T. Umeki, et al., "In-line phase sensitive amplifier based on PPLN waveguides," Opt. Express 21, 12077-12084 (2013).

[7] F. Da Ros, et al., "Phase regeneration of DPSK signals in a silicon waveguide with reverse-biased p-i-n junction,"' Opt. Express 22, 5029-5036 (2014).

[8] Y. Zhang, et al., "Phase-sensitive amplification in silicon photonic crystal waveguides," Opt. Lett. 39, 363-366 (2014).

[9] M. Ettabib, et al., "PSA-based phase regeneration of dpsk signals in a silicon germanium waveguide," in Proc. ECOC 2015, paper We3.6.5.

[10] M. Pu et al., "AlGaAs-on-insulator nanowire with $750 \mathrm{~nm}$ FWM bandwidth, $-9 \mathrm{~dB} \mathrm{CW}$ conversion efficiency, and ultrafast operation enabling record Tbaud wavelength conversion," Proc. OFC 2015, paper Th5A3.

[11] M. Pu et al., "Ultra-low-loss inverted taper coupler for silicon-oninsulator ridge waveguide," Opt. Commun., 283, 3678-3682 (2010). 